\title{
Cinética de fósforo com modelos matemáticos em ovinos adultos
}

\author{
Helder Louvandini( ${ }^{(1)}$ e Dorinha Miriam Silber Schmidt Vitti ${ }^{(2)}$
}

(1)Universidade de Brasília, Fac. de Agronomia e Medicina Veterinária, Caixa Postal 04508, CEP 70901-970 Brasília, DF. E-mail: hlouvand@unb.br (2) Centro de Energia Nuclear na Agricultura, Caixa Postal 96, CEP 13400-970 Piracicaba, SP. E-mail: vitti@cena.usp.br

\begin{abstract}
Resumo - O objetivo deste trabalho foi avaliar, por meio de modelos matemáticos, o metabolismo de fósforo $(\mathrm{P})$ em ovinos adultos suplementados com teores crescentes de farinha de ossos calcinados com $0,1,2 \mathrm{e} 3 \mathrm{~g}$ de $\mathrm{P}$ por animal por dia. Esses valores representaram diferentes tratamentos, adicionados à dieta basal com $225 \mathrm{~g}$ de concentrado e feno ad libitum. Foram utilizados 16 ovinos, Suffolk, com peso vivo de $38,2 \pm 4,35 \mathrm{~kg}$ e idade média de 18 meses, mantidos em gaiolas individuais. Após 21 dias, 7,4 MBq do radiofósforo $\left({ }^{32} \mathrm{P}\right)$ foram injetados em cada ovino, e foram coletados sangue, fezes e urina por oito dias, a fim de determinar o fluxo de P entre três compartimentos: trato gastrintestinal, compartimento central (sangue) e tecidos (moles e ósseo). Houve relação linear positiva entre o P consumido e o P absorvido, excretado nas fezes, urina e retenção. A perda endógena fecal de $\mathrm{P}$ foi exponencial com o aumento de $\mathrm{P}$ da dieta. A elevação do teor de $\mathrm{P}$ da dieta interfere nas trocas do mineral para o trato gastrintestinal e urinário, o que indica que $0,82 \mathrm{~g}$ de $\mathrm{P}$ por dia são suficientes para mantença dessa categoria animal.
\end{abstract}

Termos para indexação: diluição isotópica, mineral, modelagem.

\section{Phosphorus kinetics using mathematical models for adult sheep}

\begin{abstract}
An experiment was carried out to measure the phosphorus (P) flows in adult sheep by mathematical model. The work was conducted with 16 Suffolk sheep, live weight of $38.2 \pm 4.35 \mathrm{~kg}$ and 18 -month aged, kept in individual cage to determine the effect of phosphorus $(\mathrm{P})$ intake by adding bone meal $(0,1,2$ and $3 \mathrm{~g} \mathrm{P}$ per animal per day) in the basal diet (225 g the concentrate and hay ad libitum). After 21 days, the lambs were injected with 7.4 MBq of ${ }^{32} \mathrm{P}$ to trace the flows of $\mathrm{P}$ in the three compartments gastrointestinal tract, central (blood) and tissues (soft and bone). There was a positive relationship between $\mathrm{P}$ intake and $\mathrm{P}$ absorbed, $\mathrm{P}$ faeces, $\mathrm{P}$ urinary and $\mathrm{P}$ retained. Fecal endogenous loss of the $\mathrm{P}$ was exponentially related to $\mathrm{P}$ intake. The high levels of $\mathrm{P}$ on diet affected the exchanges between gastrointestinal and urinary tract. Results showed that $0.82 \mathrm{~g}$ of the P per day was enough for maintenance of the experimental animals.
\end{abstract}

Index terms: isotopic dilution, minerals, modeling.

\section{Introdução}

A quantificação do metabolismo animal e o conhecimento sobre o fluxo de nutrientes com a finalidade de melhor compreensão da eficiência entre tecidos, órgãos, animais e suas categorias são essenciais para nutrição animal (Baldwin, 1995). A importância do fósforo $(\mathrm{P})$ na manutenção das funções vitais não apenas na composição de estruturas, mas também participando de reações bioquímicas no organismo animal é conhecida há muito tempo. No entanto, alguns aspectos relativos ao metabolismo do $\mathrm{P}$ nos ruminantes ainda permanecem controversos como, por exemplo, a determinação da sua disponibilidade biológica e o coeficiente de absorção verdadeiro, os quais são de grande valia no conhecimento do aproveitamento do mineral fornecido na dieta e no cálculo das necessidades (Bravo et al., 2003a, 2003b).

Nos últimos anos, tem havido grande interesse no estudo do metabolismo de $\mathrm{P}$, não apenas com finalidade nutricional para o ruminante, mas com objetivo de diminuir a contaminação do ambiente, decorrente da intensificação da produção destes animais, uma vez que seus excrementos representam importante fonte de contaminação, com resíduos ricos em $\mathrm{P}$ e outras substâncias que são depositadas (Tamminga, 2003).

A técnica da diluição isotópica aliada à modelagem representa importante ferramenta no avanço da compreensão do metabolismo de $\mathrm{P}$ em ruminantes. Segundo Vitti et al. (2006), em ovinos, alguns modelos têm sido descritos e se diferenciam principalmente quanto ao número de compartimentos e aos fluxos estabelecidos entre estes. 
O modelo proposto por Aubert \& Milhaud (1960) foi desenvolvido para estudos de metabolismo de cálcio em humanos. Nesse modelo, foram estabelecidos três compartimentos, plasma, fezes e urina, de acordo com a radioatividade excretada acumulada nas fezes e urina a qualquer tempo. Embora este modelo não tenha sido desenvolvido recentemente, sua vantagem é a de não haver necessidade de o abate dos animais, para obtenção dos dados. No modelo adotado o compartimento central, sangue, está intimamente ligado aos outros dois compartimentos com intercâmbio bidirecional de acordo com o fluxo, respectivamente, para o trato gastrintestinal e tecidos. A entrada de P para o sistema ocorre via ingestão, e a eliminação pelas fezes e urina.

O objetivo deste trabalho foi avaliar, por meio de modelo matemático, os fluxos de $\mathrm{P}$ e seu metabolismo em ovinos adultos suplementados com teores crescentes de $\mathrm{P}$ oriundos da farinha de ossos calcinados e determinar a exigência de manutenção dos animais.

\section{Material e Métodos}

O trabalho foi executado no Laboratório de Nutrição Animal, do Centro de Energia Nuclear na Agricultura (Cena), da Universidade de São Paulo, Campus Piracicaba. O modelo matemático proposto utilizou resultados da pesquisa sobre a perda endógena fecal em ovinos com auxílio do radiofósforo $\left({ }^{32} \mathrm{P}\right)$ pela técnica de diluição isotópica (Louvandini \& Vitti, 1996). Foram utilizados 16 ovinos inteiros da raça Suffolk, com idade aproximada de 18 meses e peso médio inicial de $38,2 \pm 4,35 \mathrm{~kg}$. Os animais foram divididos em quatro tratamentos com quatro repetições.

Os tratamentos foram constituídos de quantidades crescentes de farinha de ossos $(0,00,8,61,17,23 \mathrm{e}$ $25,83 \mathrm{~g}$ ) adicionados ao concentrado com a finalidade de fornecer $0,1,2$ e $3 \mathrm{~g}$ de $\mathrm{P}$ por dia, respectivamente. A composição do concentrado foi de $200 \mathrm{~g}$ de farinha de mandioca, $15 \mathrm{~g}$ de uréia e $10 \mathrm{~g}$ de uma mistura com micronutrientes $\left(0,009\right.$ de $\mathrm{KI} ; 0,0008$ de $\mathrm{CoSO}_{4} ; 0,03$ de $\mathrm{CuSO}_{4} ; 1,61$ de $\mathrm{MgO} ; 3,00$ de $\mathrm{NaCl} ; 0,32$ de $\mathrm{ZnSO}_{4}$; 0,148 de $\mathrm{MnSO}_{4} ; 0,457$ de $\mathrm{FeSO}_{4}$ e 4,00 de S, em gramas por dia). Feno de Brachiaria decumbens e água foram oferecidos ad libitum. As análises bromatológicas dos alimentos foram realizadas conforme recomendações de Silva \& Queiroz (2002) (Tabela 1).

No vigésimo primeiro dia experimental, foram injetados em cada animal 7,4 MBq de ${ }^{32} \mathrm{P}$ na jugular direita. Amostras de sangue foram coletadas durante oito dias na veia jugular esquerda, cinco minutos após a injeção de ${ }^{32} \mathrm{P}$ e depois a cada 24 horas utilizando-se tubos a vácuo contendo heparina.

A determinação do P inorgânico no plasma foi realizada após centrifugação do sangue a $1.100 \mathrm{~g}$ em centrífuga Sorvall (modelo RC2B) por dez minutos e a separação das proteínas com ácido tricloroacético (10\%). $\mathrm{Na}$ determinação da atividade radioativa, foi adicionado $1 \mathrm{~mL}$ de plasma em $19 \mathrm{~mL}$ de água destilada em frascos de cintilação seguindo-se contagem por efeito Cerenkov, segundo International Atomic Energy Agency (1979).

As fezes foram maceradas em almofariz, homogeneizadas e $1 \mathrm{~g}$ pesado em cadinho para a secagem em estufa a $100^{\circ} \mathrm{C}$. Após a determinação das cinzas por calcinação em mufla a $500^{\circ} \mathrm{C}$, fez-se a digestão com $5 \mathrm{~mL}$ de ácido sulfúrico $(18 \mathrm{~N})$, e o volume total foi transferido para frascos de contagem, para determinação da radioatividade, completando-se o volume para $20 \mathrm{~mL}$. A determinação do P inorgânico nas fezes foi realizada por colorimetria, após a digestão das cinzas com ácido clorídrico, empregando-se o método do vanadato e molibdato de amônio (Sarruge \& Haag, 1974).

Amostras de urina com $30 \mathrm{~mL}$ foram digeridas a quente, em ácido clorídrico $(12 \mathrm{~N})$, secadas a $55^{\circ} \mathrm{C}$ em estufa e posteriormente queimadas a $500^{\circ} \mathrm{C}$. As cinzas foram diluídas em ácido clorídrico ( $3 \mathrm{~N}$ ) com volume ajustado em balão volumétrico de $10 \mathrm{~mL}$. A determinação do $\mathrm{P}$ inorgânico foi igual ao procedimento descrito para as fezes. Na determinação do ${ }^{32} \mathrm{P}$ presente na urina, $1 \mathrm{~mL}$ de urina foi adicionado em $19 \mathrm{~mL}$ de água destilada em frascos de cintilação.

A Figura 1 traz a representação esquemática do modelo proposto de metabolismo do $\mathrm{P}$, baseado e adaptado do modelo sugerido por Aubert \& Milhaud (1960). O modelo contém três compartimentos: trato gastrintestinal (TGI), compartimento central (sangue) representado pelo $\mathrm{P}$ lábil prontamente utilizável e os

Tabela 1. Composição bromatológica dos alimentos fornecidos aos ovinos nos diferentes tratamentos em gramas por quilograma de matéria seca.

\begin{tabular}{lrcc}
\hline Componentes & Feno & Concentrado & Farinha de ossos \\
\hline Matéria seca & 959 & 950 & 987 \\
Proteína bruta & 84 & 297 & 163 \\
Fibra bruta & 316 & 69 & - \\
Extrato etéreo & 22 & 21 & 42 \\
Cinzas & 53 & 19 & 780 \\
Fósforo & 1,7 & 0,5 & 119 \\
Cálcio & 2,4 & 1,2 & 320 \\
\hline
\end{tabular}


tecidos (moles e ósseo) analisados em conjunto. Partiu-se da premissa que não houve reentrada de ${ }^{32} \mathrm{P}$ oriundas de fontes externas. O compartimento central foi abastecido de $\mathrm{P}$ proveniente do trato gastrintestinal $\left(\mathrm{F}_{12}\right)$, pela absorção, a partir das frações dietética e endógena de origem principalmente salivar, como também do $\mathrm{P}$ do metabolismo dos tecidos (moles e ósseo) $\left(\mathrm{F}_{32}\right)$. Foi considerada como saída a perda endógena fecal (saliva, secreções dos sucos digestivos, células etc...) $\left(\mathrm{F}_{21}\right)$, $\mathrm{P}$ urinário $\left(\mathrm{F}_{20}\right)$ e do $\mathrm{P}$ retido nos tecidos $\left(\mathrm{F}_{23}\right)$. Pela diluição isotópica no equilíbrio (168 horas), as trocas no sistema permanecem constantes e a taxa de entrada é igual à taxa de saída (equação 1). Considerando-se que as frações de origem dietética $(\mathrm{Pa})$ e endógena $(\mathrm{Pd})$ compõem a $\mathrm{F}_{12}$, ao substituir-se na equação 1 , obtém-se a equação 2 .

$\mathrm{F}_{20}+\mathrm{F}_{21}+\mathrm{F}_{23}=\mathrm{F}_{12}+\mathrm{F}_{32}$

$\mathrm{F}_{20}+\mathrm{F}_{21}+\mathrm{F}_{23}=\mathrm{Pa}+\mathrm{Pd}+\mathrm{F}_{32}$

Verificando-se que Pd foi reabsorvido do TGI para o sangue, o valor das perdas totais de $\mathrm{Pa}$ a partir do compartimento central $(\mathrm{Pt})$ é igual ao total das perdas menos o valor de Pd (equação 3).

$\mathrm{Pt}=\mathrm{F}_{20}+\mathrm{F}_{21}+\mathrm{F}_{23}-\mathrm{Pd}$

em que $\mathrm{F}_{21}-\mathrm{Pd}=\mathrm{Pf}$

Ao substituir-se a equação 4 em 3, tem-se:

$\mathrm{Pt}=\mathrm{F}_{20}+\mathrm{F}_{23}+\mathrm{Pf}$

em que Pf representa as perdas endógenas fecais.

Por determinação analítica, o valor do $\mathrm{P}$ na urina foi calculado $\left(\mathrm{F}_{20}\right)$ e o do $\mathrm{P}$ endógeno fecal (Pf) foi determinado por meio das equações 6,7 e 8 .

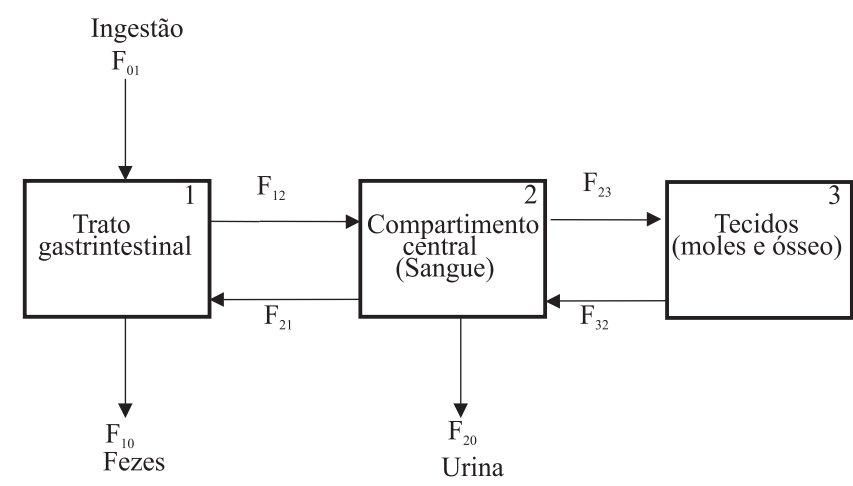

Figura 1. Modelo da cinética de fósforo em ovinos $\left(\mathrm{F}_{01}, \mathrm{P}\right.$ consumido; $\mathrm{F}_{12}$, $\mathrm{P}$ absorvido; $\mathrm{F}_{23}$, $\mathrm{P}$ do compartimento central para os tecidos; $\mathrm{F}_{32}, \mathrm{P}$ dos tecidos para sangue; $\mathrm{F}_{21}, \mathrm{P}$ do compartimento central para o trato gastrintestinal; $\mathrm{F}_{10}, \mathrm{P}$ das fezes e $\mathrm{F}_{20}$, $\mathrm{P}$ da urina).
A Atividade específica do ${ }^{32} \mathrm{P}$ no plasma ou nas fezes $=$ atividade do ${ }^{32} \mathrm{P}(\mathrm{cpm})$ de $1 \mathrm{~mL}$ de plasma ou de $1 \mathrm{~g}$ de fezes por atividade do ${ }^{32} \mathrm{P}(\mathrm{cpm})$ da solução padrão

$\% \mathrm{Pf}=100$ (Atividade específica do ${ }^{32} \mathrm{P}$ nas fezes por atividade específica do ${ }^{32} \mathrm{P}$ no plasma)

Pf total nas fezes $(\mathrm{g})=\%$ Pf $\times$ P total excretado nas fezes $(\mathrm{g})$

$\mathrm{Na}$ consideração da curva de decaimento da radioatividade do ${ }^{32} \mathrm{P}$ no plasma, foi calculado o $\mathrm{P}$ total (Pt) disponível no sangue. A curva foi dividida em duas partes: a das trocas rápidas entre o ${ }^{32} \mathrm{P}$ e o $\mathrm{P}$ estável, que corresponde ao período entre a introdução do ${ }^{32} \mathrm{P}$ até 48 horas (equações 9 e 10) e a fase das trocas lentas do ${ }^{32} \mathrm{P}$ pelo $\mathrm{P}$ estável, que ocorreu no período de três a oito dias (equação 11).

$\mathrm{Na}$ fase das trocas rápidas, o intercâmbio do ${ }^{32} \mathrm{P}$ entre o plasma e os tecidos é intenso, e grande parte do material radioativo é metabolizado, pois em torno de 48 horas após a introdução do ${ }^{32} \mathrm{P}$ no sangue, a radioatividade é reduzida a metade da dose injetada, e a maior proporção é observada nas primeiras 24 horas. Entre o compartimento central e os tecidos na fase das trocas lentas ocorre equilíbrio da atividade específica.

$\mathrm{R}=\mathrm{Ri} \mathrm{e}^{- \text {at }}$

$\mathrm{a}=0,693 / \mathrm{T}_{1 / 2}$

$\mathrm{Rs}=\mathrm{A} \mathrm{e}^{-\mathrm{at}}$

em que $\mathrm{R}$ é a radioatividade do ${ }^{32} \mathrm{P}$ no tempo $\mathrm{t}(\mathrm{cpm})$, Ri é a radioatividade inicial (cpm); a é a constante de decaimento da radioatividade; $\mathrm{T}_{1 / 2}$ é a meia-vida biológica do ${ }^{32} \mathrm{P}$ (dias); 0,693 foi calculado por dedução da equação 9 (para $\mathrm{t}=\mathrm{T}_{1 / 2}, \mathrm{R}=\mathrm{Ri} / 2$ ); Rs é o decaimento da atividade específica (relação entre ${ }^{32} \mathrm{P}$ e o P total no sangue) no tempo $\mathrm{t}(\mathrm{cpm})$; A é a atividade específica inicial (cpm). No tempo zero, a atividade específica (A) representou a relação entre a radioatividade injetada (Ri) e a massa de fósforo $(\mathrm{P})$. Assim, A = Ri/P, e, por dedução, obteve-se o valor de $\mathrm{P}$ (equação 12).

De acordo com Aubert \& Milhaud (1960), por intermédio de uma série de equações com derivadas matemáticas, as saídas de $\mathrm{P}$ do compartimento central $(\mathrm{Pt})$ foram calculadas como o produto entre a massa de $\mathrm{P}$ trocável e a constante de decaimento (equação 13).

$\mathrm{P}=\mathrm{Ri} / \mathrm{A}$

$\mathrm{Pt}=\mathrm{a} P$ 
Assim, com a determinação do valor de Pt, na equação 5, pôde-se calcular o valor de $F_{23}$, que representa a quantidade de $\mathrm{P}$ incorporada nos tecidos (moles e ósseo). $\mathrm{O}$ valor de $\mathrm{F}_{32}$ foi calculado pela equação 14 e o do $\mathrm{P}$ retido, pela diferença entre o $\mathrm{P}$ consumido $\left(\mathrm{F}_{01}\right)$ e as perdas totais nas fezes $\left(\mathrm{F}_{10}\right)$ e na urina $\left(\mathrm{F}_{20}\right)$ (equação 15$)$. No cálculo do $\mathrm{F}_{32}$, considerouse o valor do $\mathrm{F}_{23}$ e do $\mathrm{P}$ retido (equações 14 e 15 ).

$\mathrm{P}$ retido $=\mathrm{F}_{01}-\mathrm{F}_{10}-\mathrm{F}_{20}$

$\mathrm{F}_{32}=\mathrm{F}_{23}-\mathrm{P}$ retido

Considerando-se as perdas endógenas, a disponibilidade biológica (DB) (\%) foi determinada (equação 16).

$\mathrm{DB}=100\left[\left(\mathrm{~F}_{01}-\mathrm{F}_{10}+\mathrm{Pf}\right) / \mathrm{F}_{01}\right]$

A absorção real em $g$ por dia $(\mathrm{Pa})$ foi calculada pela diferença entre o P consumido ( $\mathrm{g}$ ) e $\mathrm{P}$ excretado nas fezes $(\mathrm{g})$, tendo-se descontado o valor da fração endógena fecal $(\mathrm{g})$ de acordo com a equação 17. A absorção real de $\mathrm{P}(\mathrm{Pa})$ difere do $\mathrm{P}$ do TGI para o compartimento central $\left(\mathrm{F}_{12}\right)$ pelo fato de a absorção, no caso de $\mathrm{F}_{12}$, levar em consideração as frações absorvidas de $\mathrm{P}$ de origem endógena $(\mathrm{Pd})$ e alimentar $(\mathrm{Pa})$ que chegam ao trato digestivo, enquanto a absorção real mede o valor do $\mathrm{P}$ absorvido de origem alimentar.

$\mathrm{Pa}=\mathrm{P}_{01}-\left(\mathrm{P}_{10}-\mathrm{Pf}\right)$

O delineamento experimental utilizado foi o de blocos ao acaso com dois blocos e quatro tratamentos (teores de P na dieta). Nas variáveis estudadas foram realizadas análises de variâncias com estudos de regressões polinomiais com o uso do SAS (SAS Institute, 2000), considerando $5 \%$ o nível de probabilidade.

\section{Resultados e Discussão}

Valores referentes ao consumo de matéria seca, fluxos e as variáveis relacionadas à cinética de $\mathrm{P}$ nos ovinos alimentados com teores crescentes de farinha de ossos calcinados na dieta estão apresentados na Tabela 2. Não houve diferença significativa no consumo de matéria seca entre os ovinos dos diferentes tratamentos. Quanto às variáveis $\mathrm{P}$ absorvido da dieta, $\mathrm{P}$ nas fezes, $\mathrm{P}$ na urina, $\mathrm{P}$ da dieta nas fezes, e $\mathrm{P}$ retido, houve relação linear positiva com aumento do $\mathrm{P}$ na dieta, com exceção do $\mathrm{P}$ endógeno nas fezes, cujo comportamento foi exponencial. Para os dados da disponibilidade biológica, e os fluxos do trato gastrintestinal para o compartimento central $\left(\mathrm{F}_{12}\right), \mathrm{P}$ do compartimento central para o trato gastrintestinal $\left(\mathrm{F}_{21}\right)$ e dos tecidos para o compartimento central $\left(\mathrm{F}_{32}\right)$ e o sentido inverso $\left(F_{23}\right)$ não foram significativos.

Trabalhos como os de Bravo et al. (2003a, 2003b) e Dias et al. (2007) demonstraram que o aumento do teor de $\mathrm{P}$ da dieta em ovinos eleva sua absorção, conforme observado neste trabalho $\left(\mathrm{Pa}=0,73 \mathrm{~F}_{01}+0,10, \mathrm{R}^{2}=0,86\right.$, $\mathrm{p}=0,0001)$ como também sua excreção pelas fezes $\left(F_{10}=0,47 F_{01}+0,45, R^{2}=0,72, p=0,001\right)$, já que esta é a rota preferencial de eliminação deste mineral por parte dos ruminantes. $\mathrm{O}$ comportamento exponencial da perda endógena fecal com o P consumido $\left(\mathrm{Pef}=1,68\left(1-\mathrm{e}^{-0,43 \mathrm{~F}_{01}}\right)\right.$ denota que houve aumento expressivo do tratamento 0 para os demais, mas que entre eles $(1,2$ e 3$)$ a elevação foi menor, o que indica estabilização dessa via de excreção.

Tabela 2. Consumo de matéria seca, fluxo de fósforo e disponibilidade biológica em ovinos adultos alimentados com níveis crescentes de fósforo na dieta.

\begin{tabular}{|c|c|c|c|c|c|c|c|}
\hline \multirow[t]{2}{*}{ Variáveis $\left(\mathrm{g} \mathrm{dia}^{-1}\right)$} & \multicolumn{4}{|c|}{ Tratamentos (gramas de P por dia) } & \multirow[t]{2}{*}{ Regressão } & \multirow[t]{2}{*}{$\mathrm{R}^{2}$} & \multirow[t]{2}{*}{$\mathrm{p}$} \\
\hline & 0 & 1 & 2 & 3 & & & \\
\hline $\mathrm{P}$ consumido $\left(\mathrm{F}_{01}\right)$ & 1,77 & 2,68 & 3,70 & 4,51 & & & \\
\hline Consumo de matéria seca & 1090 & 1090 & 1150 & 1130 & - & & ns \\
\hline $\mathrm{P}$ dieta absorvido (Pda) & 1,24 & 2,34 & 2,72 & 3,37 & $\mathrm{Pda}=0,73 \mathrm{~F}_{01}+0,10$ & 0,86 & 0,0001 \\
\hline$P$ fezes $\left(F_{10}\right)$ & 1,20 & 1,68 & 2,42 & 2,48 & $\mathrm{~F}_{10}=0,47 \mathrm{~F}_{01}+0,45$ & 0,72 & 0,0001 \\
\hline$P$ urina $\left(\mathrm{F}_{20}\right)$ & 0,02 & 0,02 & 0,06 & 0,06 & $\mathrm{~F}_{20}=0,021 \mathrm{~F}_{01}-0,027$ & 0,33 & 0,0191 \\
\hline $\mathrm{P}$ endógeno fezes (Pef) & 0,67 & 1,33 & 1,45 & 1,34 & Pef $=1,68\left(1-\mathrm{e}^{-0,43 \mathrm{~F} 01}\right)$ & 0,96 & 0,0001 \\
\hline$P$ dieta nas fezes (Pdf) & 0,53 & 0,34 & 0,98 & 1,14 & $\mathrm{Pdf}=0,26 \mathrm{~F}_{01}-0,09$ & 0,44 & 0,0050 \\
\hline Disponibilidade biológica (DB) \% & 0,72 & 0,87 & 0,74 & 0,75 & - & & ns \\
\hline $\mathrm{P}$ do compartimento central $(\mathrm{Pt})$ & 5,56 & 8,62 & 8,77 & 19,04 & $\mathrm{Pt}=3,58 \mathrm{~F}_{01}-0,83$ & 0,22 & 0,0685 \\
\hline$P$ gastrintestinal para compartimento central $\left(F_{12}\right)$ & 4,03 & 12,68 & 10,03 & 8,89 & - & & ns \\
\hline$P$ do compartimento central para gastrintestinal $\left(\mathrm{F}_{21}\right)$ & 3,46 & 11,66 & 8,75 & 6,86 & - & & ns \\
\hline P do compartimento central para tecidos $\left(\mathrm{F}_{23}\right)$ & 4,87 & 7,27 & 7,26 & 17,64 & $\mathrm{~F}_{23}=3,35 \mathrm{~F}_{01}-1,34$ & 0,19 & 0,0875 \\
\hline $\mathrm{P}$ dos tecidos para compartimento central $\left(\mathrm{F}_{32}\right)$ & 4,32 & 6,28 & 6,04 & 15,67 & D. $\quad-$ & & ns \\
\hline P retido & 0,55 & 0,99 & 1,22 & 1,97 & $P$ retido $=0,51 \mathrm{~F}_{01}-0,42$ & 0,76 & 0,0001 \\
\hline
\end{tabular}

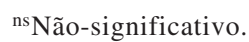


A relação linear positiva determinada entre o $\mathrm{P}$ consumido e o $\mathrm{P}$ na urina $\left(\mathrm{F}_{20}=0,021 \mathrm{~F}_{01}-0,027\right.$, $\left.\mathrm{R}^{2}=0,33, \mathrm{p}=0,0191\right)$ está associada à estabilização da perda endógena fecal, o que incrementa a rota urinária que é secundária a fecal e geralmente associada ao excesso do mineral ingerido, com teores elevados no plasma acima de 2 a $3 \mathrm{mmol} \mathrm{L}^{-1}$, ou então tipo de alimento ingerido, pois quanto maior o teor de fibra efetiva ingerida maior produção de saliva e excreção de $P$ pelas fezes em detrimento da urina e vice-versa (Bravo et al., 2003b). Neste experimento, o estímulo da ruminação e salivação foram garantidos pela presença das fibras longas do feno na dieta e houve retenção positiva de $\mathrm{P}$ em todos os tratamentos. $\mathrm{O}$ aumento de $\mathrm{P}$ na urina é mais uma indicação de que os teores de $\mathrm{P}$ utilizados seriam adequados para a necessidade dos ovinos, tendo a urina como uma rota de controle da homeostase do mineral.

À primeira vista, parece contraditória a situação da perda endógena fecal ter ritmo de excreção menor e de característica exponencial enquanto o $\mathrm{P}$ nas fezes linear. No entanto, nas fezes verifica-se contribuição do $\mathrm{P}$ da dieta, que não foi absorvido, o que contribuiu para tal efeito. Em razão da diminuição do $\mathrm{P}$ endógeno, o P nas fezes de origem alimentar chegou a $46 \%$ do total excretado no tratamento de $3 \mathrm{~g}$, quantidade expressiva, que deve ser descontada do que foi fornecido e não utilizado pelo animal. Nessa etapa, pode haver desperdício de $\mathrm{P}$ e, conseqüentemente, maior contaminação ambiental.

Os coeficientes de disponibilidade biológica do $\mathrm{P}$ apresentaram pouca variação, tendo valor médio de 0,77 entre todos os tratamentos, o que está acima do preconizado para ruminantes, de 0,73 pelo Agricultural Research Council (1994), 0,65 em forragens, e 0,70 em concentrados, pelo National Research Council (2001) e 0,72, por Bravo et al. (2003a). Neste trabalho, a disponibilidade do mineral foi avaliada na dieta total (forragem, concentrado e farinha de ossos calcinados). Além do tipo de alimento, a fonte de $\mathrm{P}$ usada exerce papel determinante na absorção deste elemento e a farinha de ossos tem alta disponibilidade de $\mathrm{P}$, como foi verificado.

O fluxo de $\mathrm{P}$, do compartimento central para os tecidos (moles e ósseo), iniciou-se em 4,87 g por dia, chegando até $17,64 \mathrm{~g}$ por dia para o tratamento 3. Segundo Bueno \& Vitti (1998), há diferenças entre as taxas metabólicas entre os tecidos, em que os ossos possuem dinâmica de incorporação mais lenta, com resposta mais tardia ao teor de $\mathrm{P}$ da dieta, e os tecidos moles possuem dinâmica de incorporação mais rápida e dependente do $\mathrm{P}$ da dieta imediata. Neste trabalho, em virtude de os tecidos moles e os ossos serem considerados como um único compartimento, torna-se difícil afirmar qual foi o local de maior retenção do $\mathrm{P}$, sendo esta uma das limitações do modelo.

A retenção consolida o fato de haver maior saída de $\mathrm{P}$ do compartimento central para os tecidos, à medida que o $\mathrm{P}$ da dieta se eleva $\left(\mathrm{P}\right.$ retido $=0,51 \mathrm{~F}_{01}-0,42$, $\left.\mathrm{R}^{2}=0,76, \mathrm{p}=0,0001\right)$. Por meio da equação descrita, na retenção zero de $\mathrm{P}$ (mantença), o $\mathrm{P}$ consumido seria de 0,82 g por dia e para Louvandini \& Vitti (1996) seria de 0,85 g por dia. Segundo o Agricultural Research Council (1994), para a mantença de ovino de $35 \mathrm{~kg}$ de peso vivo, a quantidade de $\mathrm{P}$ necessária seria entre 0,6 a $0,8 \mathrm{~g}$ por dia. No trabalho realizado por Vitti et al. (2005), utilizando-se dados de 98 ovinos da raça Suffolk, aplicando o modelo de Vitti et al. (2000) com quatro compartimentos (separação entre tecidos moles e ósseo) e com consumo de $\mathrm{P}$ variando de 1,45 a 7,96 g por dia, o valor para mantença indicado foi de $0,99 \mathrm{~g}$ por dia, estando próximo ao valor calculado neste trabalho.

No entanto, utilizando-se a equação preconizada pelo National Research Council (2006), em recente revisão das exigências nutricionais para ovinos, $O$ valor de $P$ para mantença de animais, com consumo similar ao deste experimento, seria de $1,64 \mathrm{~g}$ por dia, com aproximadamente o dobro do valor obtido. Essa diferença pode ser devida ao valor mais baixo para o coeficiente de absorção que o National Research Council indica $(0,60)$. Deve-se lembrar que neste experimento, com o uso da técnica de diluição isotópica, determinou-se o coeficiente de absorção real. Mesmo com a adoção do coeficiente de absorção de 0,77 , na equação descrita no National Research Council, a exigência de mantença ainda é superior (1,28 g por dia). Fica evidente que as diferenças entre as recomendações ainda permanecem bastante discrepantes e merecem mais estudos, uma vez que o P é o terceiro nutriente mais caro na formulação de rações e considerado o mais poluente.

\section{Conclusões}

1. A elevação do teor de fósforo da dieta interfere nas trocas do mineral para o trato gastrintestinal e urinário, e promove maior retenção nos tecidos moles e ósseo. 
2. O modelo matemático utilizado é capaz de representar a retenção de fósforo, o que indica que $0,82 \mathrm{~g}$ de fósforo por dia é suficiente para a mantença da categoria animal estudada.

\section{Referências}

AGRICULTURAL RESEARCH COUNCIL. The nutrient requirement of ruminant livestock. Farnham Royal: $\mathrm{CAB}$ International, 1994. 351p.

AUBERT, J.P.; MILHAUD, G. Méthod de mesure dês principales voies du métabolisme calcique chez l'homme. Biochimica et Biophysica Acta, v.39, p.122-139, 1960.

BALDWIN, R.L. Modelling ruminant digestion and metabolism. New York: Champman \& Hall, 1995. 578p.

BRAVO, D.; SAUVANT, D.; BOGAERT, C.; MESCHY, F. II: Quantitative aspects of phosphorus absorption in ruminants. Reproduction and Nutrition Development, v.43, p.271-284, 2003a.

BRAVO, D.; SAUVANT, D.; BOGAERT, C.; MESCHY, F. III: Quantitative aspects of phosphorus excretion in ruminants. Reproduction and Nutrition Development, v.43, p.285-300, 2003 b.

BUENO, M.S.; VITTI, D.M.S.S. Cinética de fósforo em tecidos de caprinos. Pesquisa Agropecuária Brasileira, v.33, p.1609-1615, 1998.

DIAS, R.S.; KEBREAB, E.; VITTI, D.M.S.S.; PORTILHO, F.P.; LOUVANDINI, H.; FRANCE, J. Phosphorus kinetics in lambs fed differents levels of dicalcium phosphate. Journal of Agricultural Science, v.145, p.509-516, 2007.

INTERNATIONAL ATOMIC ENERGY AGENCY. Laboratory training manual on the use of nuclear techniques in animal research. Vienna: IAEA, 1979. 299p. (Technical report series, 193).
LOUVANDINI, H.; VITTI, D.M.S.S. Phosphorus metabolism and estimation of phosphorus requirements for sheep. Scientia Agricola, v.53, p.184-189, 1996.

NATIONAL RESEARCH COUNCIL. Nutrient requirements of dairy cattle. 7.ed. Washington: National Academic Press, 2001.450p.

NATIONAL RESEARCH COUNCIL. Nutrient requirements of small ruminants: sheep, goats, cervids, and New World camelids. Washington: National Academic Press, 2006. 362p.

SARRUGE, J.R.; HAAG, H.P. Determinação colorimétrica do fósforo. In: SARRUGE, J.R.; HAAG, H.P. Análises químicas em plantas. Piracicaba: Esalq, 1974. p.6-58.

SAS INSTITUTE. The SAS system for windows. Release 8.01. Cary: SAS Institute, 2000.

SILVA, D.J.; QUEIROZ, A.C. Análise de alimentos: métodos químicos e biológicos. 3.ed. Viçosa: UFV, 2002. 235p.

TAMMINGA, S. Pollution due to nutrient losses and its control in European animal production. Livestock Production Science, v.84, p.101-111, 2003.

VITTI, D.M.S.S.; KEBREAB, E.; LOPES, J.B.; ABDALLA, A.L.; CARVALHOS, F. F.R. de; RESENDE, K.T. de; CROMPTON, L.A.; FRANCE, J. A kinetic model of phosphorus metabolism in growing goats. Journal of Animal Science, v.78, p.2706-2712, 2000.

VITTI, D.M.S S.; KEBREAB, E.; LOPES, J.B.; ABDALLA, A.L.; FRANCE, J. Effects of dietary supplementation on phosphorus metabolism in sheep. Journal of Animal and Veterinary Advances, v.4, p.356-359, 2005

VITTI, D.M.S.S.; ROQUE, A.P.; DIAS, R.S.; LOPES, J.B.; BUENO, I.C.S.; BUENO, M.S.; NOZELLA, E.F. Metabolismo de cálcio em ovinos em crescimento sob suplementação com diferentes fontes de cálcio: aplicação e comparação de dois modelos matemáticos. Revista Brasileira de Zootecnia, v.35, p.2487-2495, 2006.

Recebido em 21 de junho de 2007 e aprovado em 20 de setembro de 2007 